\title{
Hábitos alimenticios y sus efectos en la salud de los estudiantes de la Facultad de Ciencias Económicas de la Universidad de Cartagena ${ }^{1}$
}

\author{
Astrid Carolina Arroyo Pérez², Dayana Gutiérrez Puerta ${ }^{3}$, Alexandra Narváez Castillo ${ }^{4}$ \\ Paula Andrea Ramos Lozano ${ }^{5}$, Francisco Javier Maza Ávila ${ }^{6}$ \\ Universidad de Cartagena - Colombia
}

\section{ACCESo $\boldsymbol{\sigma}$ ABIerto}

Para citaciones: Arroyo, A., Gutierrez, D., Narváez, A., Ramos, P., Maza, F. (2020).

Hábitos alimenticios y sus efectos en la salud de los estudiantes de la Facultad de Ciencias Económicas de la Universidad de Cartagena. Revista de jóvenes investigadores Ad Valorem, 3(2), 100-114

Editor: Bernardo Romero Torres. Universidad de Cartagena-Colombia.

Tipología IBN Publindex: Artículo de investigación científica y tecnológica
Copyright: (C) 2020. Arroyo, A., Gutierrez, D., Narváez, A., Ramos, P., Maza, F. Este es un artículo de acceso abierto, distribuido bajo los términos de la licencia Creative Commons Atribución-NoComercial-SinDerivados 4.0 la cual permite el uso sin restricciones, distribución y reproducción en cualquier medio, siempre y cuando que el original, el autor $y$ la fuente sean acreditados.

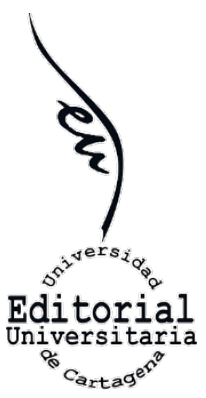

\section{RESUMEN}

Este artículo tiene como objetivo analizar los hábitos alimenticios de los estudiantes de la Facultad de Ciencias Económicas de la Universidad de Cartagena, así como la percepción que tienen sobre la incidencia de dichos hábitos en su salud, como base para la formulación de recomendaciones que contribuyan a su mejora. Para indagar los hábitos alimenticios y las percepciones de incidencia en la salud, se aplicó un cuestionario, mediante la técnica encuesta, a una muestra piloto de 100 estudiantes de la institución; asimismo, se levantó la información de los establecimientos que ofertan alimentos tanto al interior de la sede Piedra de Bolívar, como en su área de influencia. Los resultados arrojaron que la mayor parte de los estudiantes adquieren los alimentos al interior de la universidad. De igual forma, consideran que los hábitos alimenticios si están relacionados directamente con su salud, en especial, con enfermedades asociadas a una mala alimentación. En cuanto a la oferta, se evidenció que la mayoría de los establecimientos no ofertan alimentos saludables, mientras que aquellos pocos que lo hacen, presentan una baja demanda; asimismo, entre los factores que definen la elección de los alimentos por parte de los estudiantes, se encuentran el precio y el sabor. Finalmente, se proporcionaron recomendaciones para mejorar los hábitos alimenticios y la salud, entre éstas, variar los alimentos ingeridos semanalmente, no depender solo de los ofertados en la institución y recurrir a otras alternativas, tales como llevar alimentos preparados que aporten más contenido nutricional a su dieta diaria.

Palabras clave: Efectos en la Salud; Hábitos Alimenticios; Percepciones.

\footnotetext{
1 Este documento participó, en la modalidad Proyectos con Resultados, en las XI Jornadas de Investigación de la Facultad de Ciencias Económicas de la Universidad de Cartagena, año 2020.

2 Estudiante del programa de Administración Industrial de la Universidad de Cartagena, Colombia. E-mail: aarroyop@unicartagena.edu.co

3 Estudiante del programa de Administración Industrial de la Universidad de Cartagena, Colombia. E-mail: dgutierrezp@unicartagena.edu.co

4 Estudiante del programa de Administración Industrial de la Universidad de Cartagena, Colombia. E-mail: anarvaezc1@unicartagena.edu.co

${ }^{5}$ Estudiante del programa de Administración Industrial de la Universidad de Cartagena, Colombia. E-mail: pramos|@unicartagena.edu.co

${ }^{6}$ Doctor en Ciencias Sociales y Jurídicas, Universidad de Cádiz, España. Docente investigador adscrito al programa de Administración Industrial de la Facultad de Ciencias Económicas y al Instituto Internacional de Estudios del Caribe, Universidad de Cartagena, Colombia. Director del Grupo de Investigación en Estudios para el Desarrollo Regional GIDER-. E-mail: fmazaa@unicartagena.edu.co
} 


\title{
Eating habits and their effects on the health of students in The Faculty of Economics Sciences at the University of Cartagena
}

\begin{abstract}
This article aims to analyze the eating habits of students in the Faculty of Economics Sciences at University of Cartagena, as well as the perception they have about the impact of these habits on their health, as a basis for the formulation of recommendations that contribute to their improvement. To investigate the eating habits and perceptions of health impact, a questionnaire was applied through the survey technique to a pilot sample of 100 students of the institution, also raised information from establishments that offer food both within the Piedra de Bolivar headquarters, and in its area of influence. The results showed that most of the students purchase food inside the university. Similarly, they consider that eating habits are directly related to their health, especially with diseases associated with poor nutrition. Regarding the supply, it was shown that most of the establishments do not offer healthy food, while those few that do, present a low demand; likewise, among the factors that define the choice of food by students, are the price and the taste. Finally, recommendations were provided to improve eating habits and health, including varying the foods eaten weekly, not relying only on those offered at the institution and resorting to other alternatives, such as bringing prepared foods that provide more nutritional content to their daily diet.
\end{abstract}

Key words: Eating Habits; Health Effects; Perceptions.

\section{INTRODUCCIÓN}

Según datos de la Organización de Naciones Unidas para la Alimentación y la Agricultura -FAO, por sus siglas en inglés- en América Latina el sector agrícola aumentó en un 4\% al año 2018, pero sostiene que adquirir alimentos de buena calidad se hace cada vez más difícil para aquellas poblaciones que no cuentan con recursos suficientes para comprarlos, es decir, que en esta región, el problema no radica en la insuficiencia de alimentos, sino a las condiciones socioeconómicas que limitan el acceso a mayores estándares de vida (FAO, 2018). Hoy día es más fácil conseguir alimentos ricos en sal, grasas y carbohidratos que aquellos que se consideran saludables. Por ello, la FAO como organismo multilateral, está orientado a la búsqueda de una mayor productividad en los diferentes sectores, de tal manera que la población latinoamericana cuente con productos de calidad que puedan adicionar a su dieta habitual. Otro factor vinculado que causa daños en la alimentación son los agroquímicos tóxicos, que han elevado el índice de enfermedades crónicas no transmisibles en el continente americano. En la actualidad, 821 millones de personas alrededor del mundo sufren subalimentación crónica. Por otro lado, los niveles de obesidad están aumentando también de forma vertiginosa. Existen también países que experimentan la doble carga nutricional: El hambre y la obesidad, pues 1.900 
millones de personas padecen sobrepeso, de los cuales 672 millones son considerados obesos (OMS, 2016).

En el caso de Colombia, la última Encuesta Nacional de la Situación Nutricional en Colombia -ENSIN- realizada en el año 2015, arroja que sus habitantes poseen pésimos hábitos alimenticios. Según esta fuente, el 28\% de los niños de entre 6 meses y 5 años tienen anemia, y esa enfermedad está presente en el 11\% de los adolescentes y en el 8\% de las mujeres en edad fértil. Por su parte, 7 de cada 10 personas suelen incorporar a su dieta comida comprada en la calle y el 37\% consume golosinas todos los días. Además, el 39\% de los encuestados expresó no consumir ni productos lácteos ni frutas ni verduras a diario. 5 de cada 7 no consumen verduras y 7 de cada 10 incorporan comidas de la calle en su alimentación diaria (ICBF, 2018).

La alimentación es supremamente importante en cada una de las etapas del ciclo vital, aún más durante el periodo de la vida universitaria, crucial etapa en el desarrollo formativo de la persona, y que bien acompañado, de forma paralela, con cambios físicos, fisiológicos y psicosociales. Además, es un momento clave para la instauración de hábitos alimenticios saludables, puesto que se presentan alteraciones metabólicas que hacen necesaria la ingesta adecuada de proteínas y aportes vitamínicos (Arroyo, Gutiérrez, Narváez, Ramos y Maza, 2019).

Otro de los elementos pertinentes a la alimentación durante la adolescencia es la aparición de nuevos retos, autonomía, independencia y aumento de la responsabilidad en sus acciones, tales como qué, cómo, cuándo y dónde comer (Cervera et al., 2013; Burriel, Serrano, Vico, Milla y García, 2013), lo que impactaría en gran manera en su propio bienestar.

Los anteriores problemas asociados a malos hábitos alimenticios no son ajenos a los estudiantes de la de la Universidad de Cartagena sede Piedra de Bolívar, una institución educativa superior de carácter oficial, localizada en el sector homónimo en la ciudad de Cartagena de Indias, fundada en el año 1827 por Simón Bolívar y Francisco De Paula Santander y reconocida por ser la más antigua del Caribe colombiano y una de las mejores universidades del país (Universidad de Cartagena, 2017).

La Sede Piedra de Bolívar de la Universidad de Cartagena es la más grande de las sedes en cuanto a población se refiere, sumando un total de 4.522 estudiantes en modalidad presencial para el segundo semestre del año 2018, representando el $44,4 \%$ de la población de estudiantes de esta modalidad (Universidad de Cartagena, 2018). Cuenta con dos facultades: Ingeniería y Ciencias Económicas. A pesar de contar con la mayor población, cuenta con una limitada oferta de alimentos, considerando la cantidad de estudiantes que transitan en ésta; además, es probable que la oferta de alimentos no cumpla al 100\% con los 
estándares saludables requeridos por una población universitaria, por tanto, la repercusión que tendría sobre la población estudiantil no sería nada favorable.

Con base a lo anterior, esta investigación pretende indagar los hábitos alimenticios de los estudiantes de la Facultad de Ciencias Económicas en su quehacer cotidiano, identificando sus pautas de consumo y la posible relación con su salud, teniendo en cuenta factores como el horario de los estudiantes o la aproximación entre su vivienda y lugar de estudio, haciendo énfasis en las épocas de exámenes parciales, que llevan al estudiante a pasar mayor tiempo en la universidad para garantizar un mejor ritmo de estudio, y puede llevar a optar por alimentarse en los lugares contiguos a la institución y en periodos por fuera de los habituales. Se pretende resolver a la siguiente pregunta: ¿Cuáles son los hábitos alimenticios de los estudiantes de la Facultad de Ciencias Económicas de la Universidad de Cartagena, sede piedra de Bolívar, y cuál es la percepción que ellos tienen sobre los efectos que dichos hábitos tienen en su salud?

La presente investigación adquiere gran interés por su aporte al conocimiento en temas relacionados con el ámbito de la salud, en este caso se aborda el tema referente a los hábitos alimenticios en estudiantes universitarios ya que, por medio de este trabajo, se busca abordar, de forma detallada, los hábitos alimenticios que tienen los estudiantes universitarios de la Facultad de Ciencias Económicas de la Universidad de Cartagena, sede Piedra de Bolívar, y las causas que los conllevan a adquirirlos, así como la percepción que ellos tienen sobre la incidencia de dichos hábitos en su salud; asimismo, en el curso de la investigación se establecieron criterios que exige la metodología para la obtención de resultados confiables con el fin de formular recomendaciones que contribuyan a la aplicación de buenos hábitos alimenticios.

\section{METODOLOGÍA}

El presente proyecto investigativo se desarrolló al interior de la Universidad de Cartagena, en la sede Piedra de Bolívar, con especial atención a los estudiantes de los programas presenciales de la Facultad de Ciencias Económicas. Por su alcance y propósito, se sitúa en el marco de la investigación explicativa, pues se enfatiza en los motivos que llevan a la elección de hábitos alimenticios de los estudiantes de la Universidad de Cartagena, así como la percepción que tienen sobre la influencia de estos en su salud. Por otra parte, esta pesquisa también es de naturaleza propositiva, teniendo en cuenta que uno de los objetivos de la investigación es sugerir un conjunto de estrategias o recomendaciones, las cuales conlleven al estudiante a mantener una buena alimentación o a contribuir a unos hábitos alimenticios más saludables.

Para la recolección de datos referentes a los hábitos alimenticios, tipos de alimentos consumidos y la percepción de estos mismos en la salud, se 
implementó un cuestionario, mediante la técnica encuesta, a estudiantes de programas en modalidad presencial de la Facultad de Ciencias Económicas. Asimismo, se diseñó una lista chequeo con el propósito de analizar la oferta y calidad del servicio de alimentación brindado por los establecimientos internos y aledaños a la Universidad de Cartagena, sede Piedra de Bolívar. Ambos métodos de recolección de información fueron diseñados por el equipo investigativo, a partir de los estándares propuesto por la Organización Mundial de la Salud, y fueron aplicados durante el segundo periodo del año 2019.

El cuestionario fue aplicado a una muestra piloto de 100 estudiantes. Dicha muestra fue seleccionada mediante muestreo aleatorio estratificado, con afijación proporcional, en los distintos programas presenciales que posee la Facultad de Ciencias Económicas, como lo son: Contaduría Pública -diurna y nocturna-, Economía, Administración de Empresas - diurna y nocturna- y Administración Industrial. Al momento de ejecutar los cuestionarios, se hizo énfasis en los estudiantes que se encontraban cursando semestres en el rango de segundo a noveno, puesto que aquellos encontrados en primer semestre se consideran estudiantes en proceso de definición de sus hábitos de consumo frente a la vida universitaria, y los de último semestre cuentan con poca presencia en la sede Piedra De Bolívar, por tanto, no aportarían datos lo suficientemente fundamentados para el desarrollo de la investigación.

\section{RESULTADOS}

\subsection{La oferta alimentaria en el entorno físico de la Universidad de Cartagena}

La Universidad de Cartagena cuenta con diez establecimientos dentro y fuera de su campus, siendo tres los internos; la cafetería y dos negocios ambulantes. En ellos, su oferta alimentaria se basa mayormente en alimentos sólidos y líquidos con alto contenido en azúcar, mantecas y aceites. Por otro lado, se encuentran los establecimientos externos o aledaños a la universidad de Cartagena, de los cuales 5 ofertan comidas que contienen los aportes nutricionales necesarios o balanceados. Por último, se encuentran dos establecimientos que brindan principalmente frituras y bebidas industriales azucaradas. El siguiente mapa muestra la ubicación de los restaurantes tanto a interior como en el área de influencia de la Universidad de Cartagena (Mapa 1). 
Mapa 1. Ubicación de los restaurantes al interior y en el área de influencia de la Universidad de Cartagena sede Piedra de Bolívar

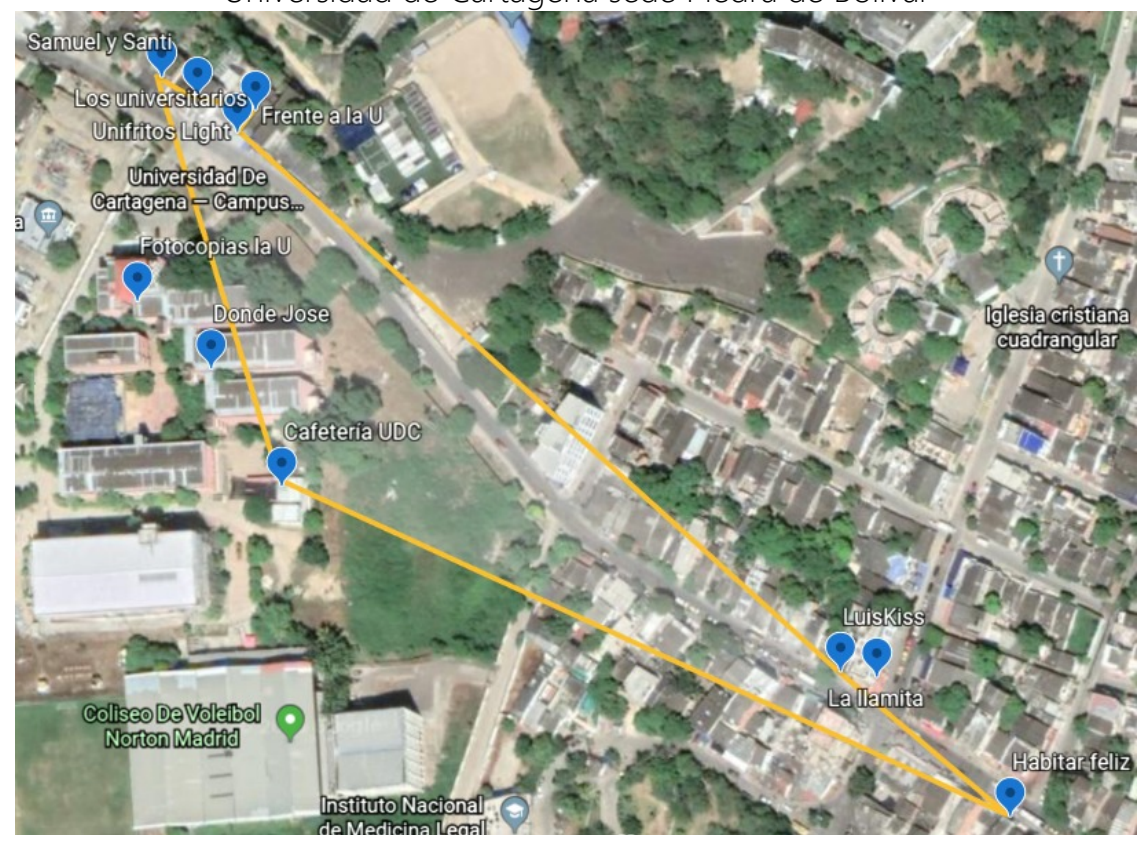

Fuente: Elaboración propia, a partir de Google Maps

En cuanto a la oferta alimentaria encontrada en los establecimientos dentro y fuera de la universidad, en un radio de 400 metros, se encontró que la mayor oferta en un $80 \%$ de los establecimientos fue las bebidas azucaradas de origen industrial, en contraste con un $40 \%$ de locales que ofertan jugos naturales. Además, se pudo establecer que el precio promedio de bebidas azucaradas es de $\$ 1.325$ y el de jugos naturales es de $\$ 2.423$. Por tanto, teniendo en cuenta la alta oferta y el bajo precio de las bebidas azucaradas resulta más tentador y posible para los estudiantes escogerlas antes que los jugos naturales.

Para el caso de las frituras, se pudo establecer que el 30\% de los locales objeto de estudio las ofertan. Aunque aparentemente es un bajo porcentaje de esta oferta, es importante considerar que es uno de los productos por los cuales se muestra mayor preferencia, considerando que su precio promedio oscila en $\$ 1.580$, mientras que los precios de un almuerzo corriente, un seco, asado, entro otros, están en promedio en \$7.000. En ese sentido, queda por sentado que, a pesar de encontrar que un almuerzo trae consigo más aportes nutritivos que una fritura, los estudiantes, consumen de este último, dado su poder adquisitivo. A esto, se le puede añadir que, según las encuestas realizadas, el 66\% de los estudiantes consumen alimentos de la oferta que se encuentra al interior de la Universidad de Cartagena, sede Piedra de Bolívar (Gráfica 2), el 67\% consumen frituras en jornadas académicas de dos a cuatro veces por semana, siendo estos los días más representativos en los resultados (Gráfica 3), por último, más del 70\% de los mismos especificó un gasto semanal en alimentos durante la semana y el 
promedio de esos valores está dado en $\$ 16.164$, por lo que se hace evidente la poca inversión que realizan en su ingesta alimentaria en la universidad.

Gráfica 2. Estudiantes que consumen alimentos en establecimiento ubicados dentro de la Universidad de Cartagena o en su área de influencia

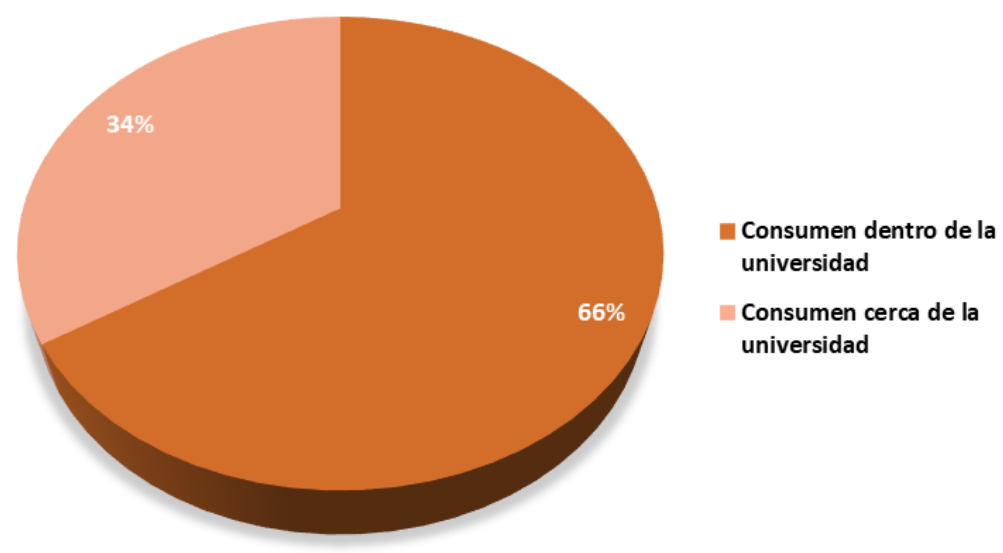

Fuente: Elaboración propia, a partir de cuestionarios aplicados a estudiantes

Gráfica 3. Consumo semanal de frituras de los estudiantes

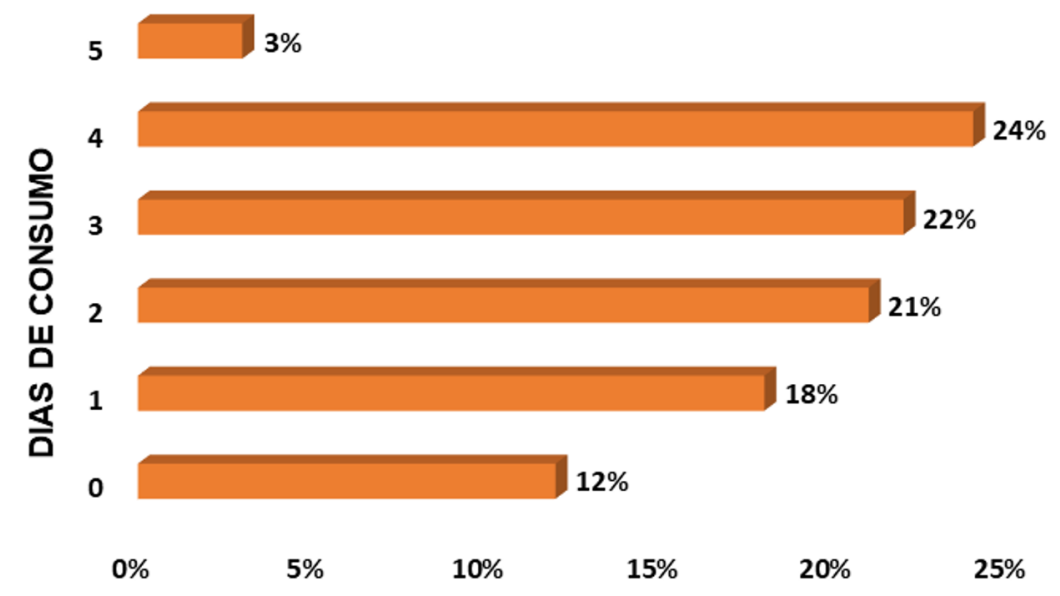

Fuente: Elaboración propia, a partir de cuestionarios aplicados a estudiantes

La oferta más limitada en el entorno está dada por las comidas rápidas, se encuentran en un solo restaurante y su consumo es mínimo, el 67\% de los encuestados afirma consumir menos de tres veces por semana este tipo de alimentos en horarios académicos (Gráfica 4). Por último, pero no menos importante, se encuentra una oferta de meriendas empaquetadas y dulces, propuesta por el $40 \%$ de los establecimientos, en ella se encuentra un promedio de precios de $\$ 1.111$ para las meriendas y $\$ 470$ para los dulces, para las cuales el mayor consumo está entre dos y tres veces por semana para el $50 \%$ de la 
población y, de ese porcentaje, menos del 30\% los consume entre comidas (Gráfico 5).

Grafica 4. Consumo semanal de comidas rápidas por parte de los estudiantes

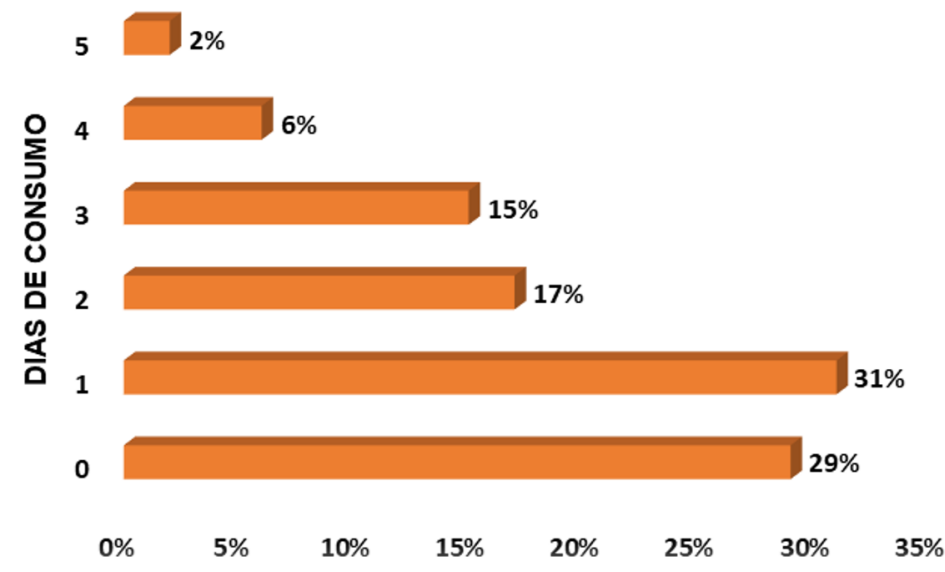

Fuente: Elaboración propia, a partir de cuestionarios aplicados a estudiantes

Grafica 5. Consumo semanal de meriendas y dulces por parte de los estudiantes

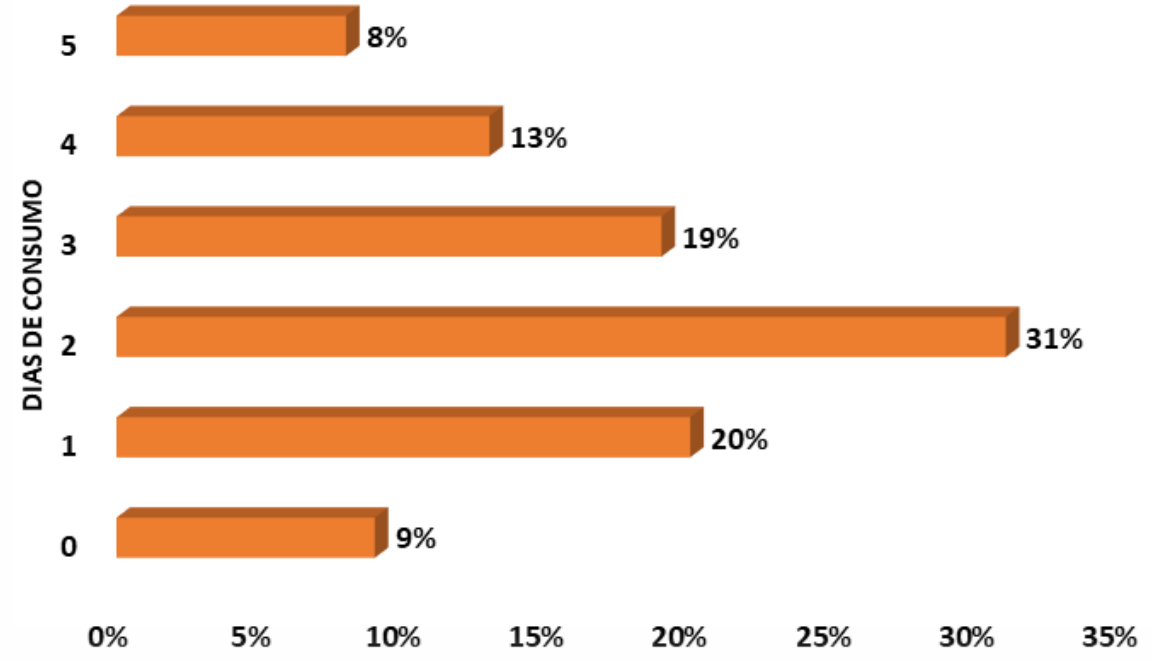

Fuente: Elaboración propia, a partir de cuestionarios aplicados a estudiantes

\subsection{Elección de consumo de alimentos de los estudiantes de la Universidad de Cartagena, sede Piedra de Bolívar}

Durante el proceso de recolección de información se preguntó a la población encuestada los motivos por los cuales elegían un alimento para su consumo. Se obtuvo como resultado que el $44 \%$ de los estudiantes eligen sus alimentos por el precio y $31 \%$ por su sabor, afirmando nuevamente que uno de los factores que más incide en la creación de buenos o malos hábitos alimenticios viene dado por 
los recursos económicos disponibles. El contenido nutricional de los alimentos a consumir es evaluado y tenido en cuenta por un porcentaje mínimo de la población (12\%) (Grafica 6). Por otro lado, se evaluaron las razones por las que un individuo evita el consumo de algún alimento, para este respecto se obtuvo que $56 \%$ de la población lo hace porque no le gusta y, solo un $28 \%$ de la misma afirma que lo hace por cuidarse, por lo que se puede afirmar que son muy pocos los jóvenes interesados en el cuidado de su salud y en la aplicación de hábitos alimenticios saludables (Gráfica 7).

Gráfica 1. Motivos de los estudiantes para la elección de alimentos

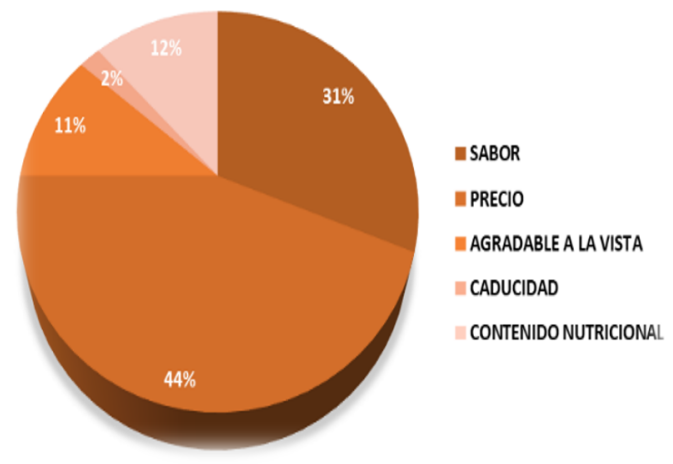

Fuente: Elaboración propia, a partir de cuestionarios aplicados a estudiantes

Gráfica 2. Motivos de los estudiantes para evitar el consumo de alimentos

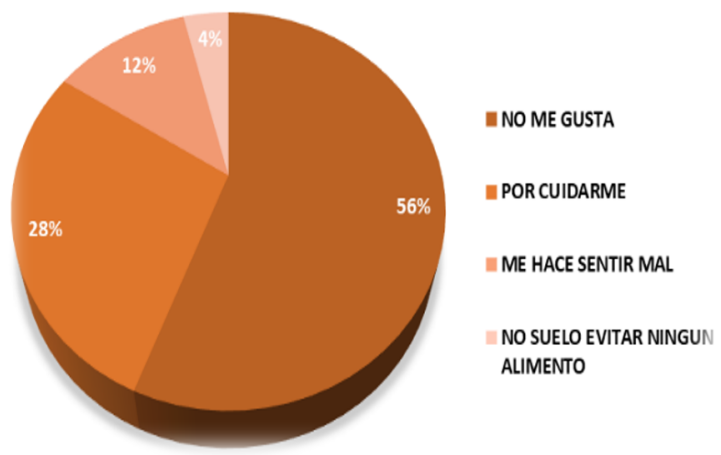

Fuente: Elaboración propia, a partir de cuestionarios aplicados a estudiantes

\subsection{Hábitos alimenticios y percepción de incidencia en la salud}

Los estudiantes universitarios, al ser jóvenes en su mayoría, vulnerables en el ámbito nutricional, debido a factores como los horarios, componentes económicos, carga académica, limitación de tiempo u oferta; por ende, sus hábitos alimenticios no suelen ser los más adecuados, ya que no se encuentran dentro de los estándares recomendados por la OMS (organización mundial de la salud), dichos estándares suelen estar expresados en una pirámide nutricional 
(Ilustración 1), la cual es una herramienta donde se muestra de manera gráfica las cantidades que se deberían consumir para ingerir los nutrientes necesarios para el día a día.

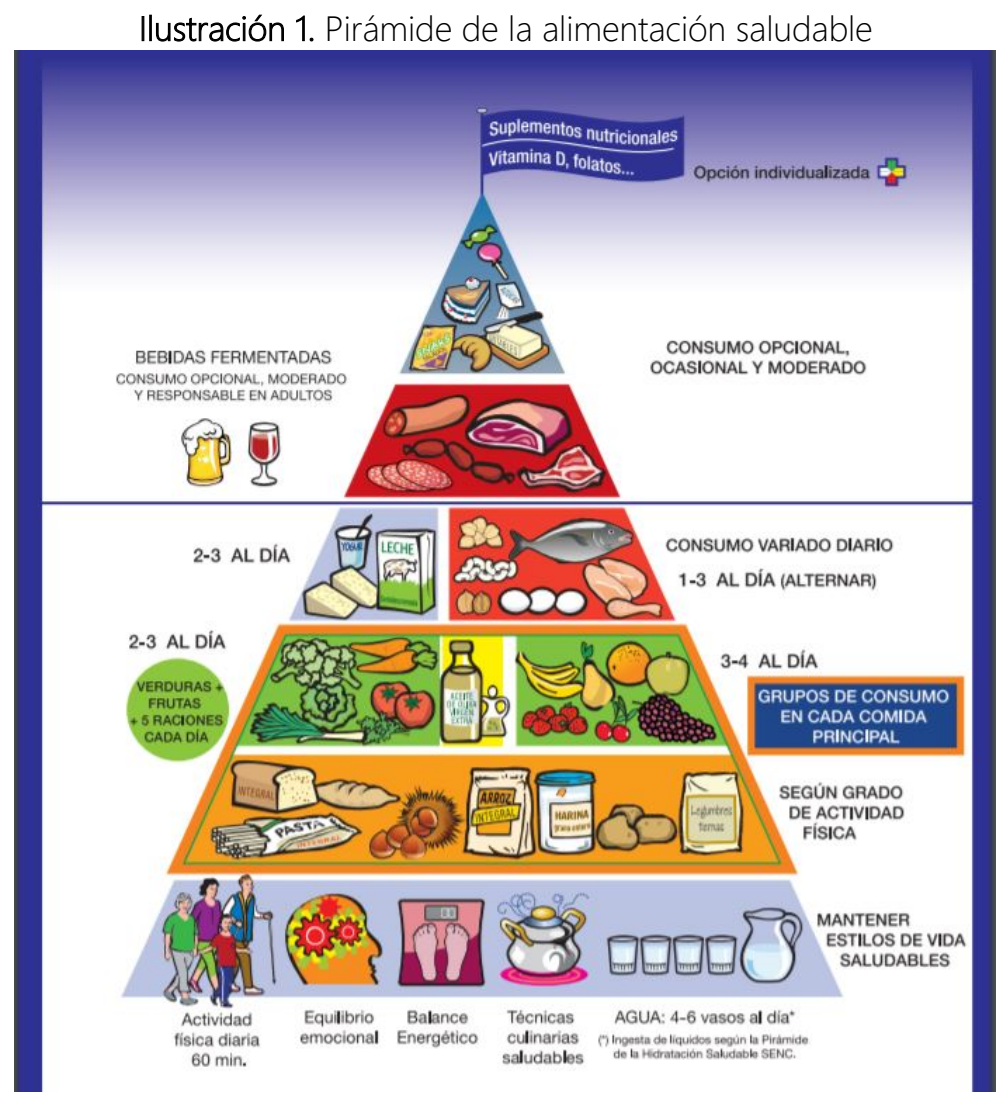

Fuente: SENC (Sociedad española de nutrición comunitaria)

La pirámide de la alimentación explica qué alimentos y en qué cantidades se deben consumir para tener una dieta equilibrada, en efecto, una persona debe consumir entre cuatro y seis raciones al día de alimentos que contengan carbohidratos, para las frutas tres porciones diarias y para las verduras dos, las proteínas requieren una ingesta de tres a cuatro raciones a la semana y, en última instancia están los dulces y/o embutidos que se deben consumir ocasionalmente.

La población estudiantil de la Facultad de Ciencias Económicas, a pesar de no contar con unos aceptables hábitos alimenticios, es baja la proporción que señala sufrir de enfermedades tales como la migraña, la cual solo se presenta en un 19\% del estudiantado y siendo la más frecuente y común, seguida de la gastritis (15\%), luego le siguen el colesterol y triglicéridos, con unos porcentajes de $4 \%$ y $2 \%$, respectivamente. Para la diabetes se tiene que ninguna de las personas encuestadas presenta dicha enfermedad. Por otro lado, el 64\% de la población no sufre de ninguna de las enfermedades ya mencionadas, siendo un porcentaje representativo, por lo cual, se puede concluir que el estudiantado cuenta con un estado de salud medianamente satisfactorio (Gráfico 8). 
Gráfica 8. Porcentaje de estudiantes que sufren de enfermedades relacionadas con la alimentación

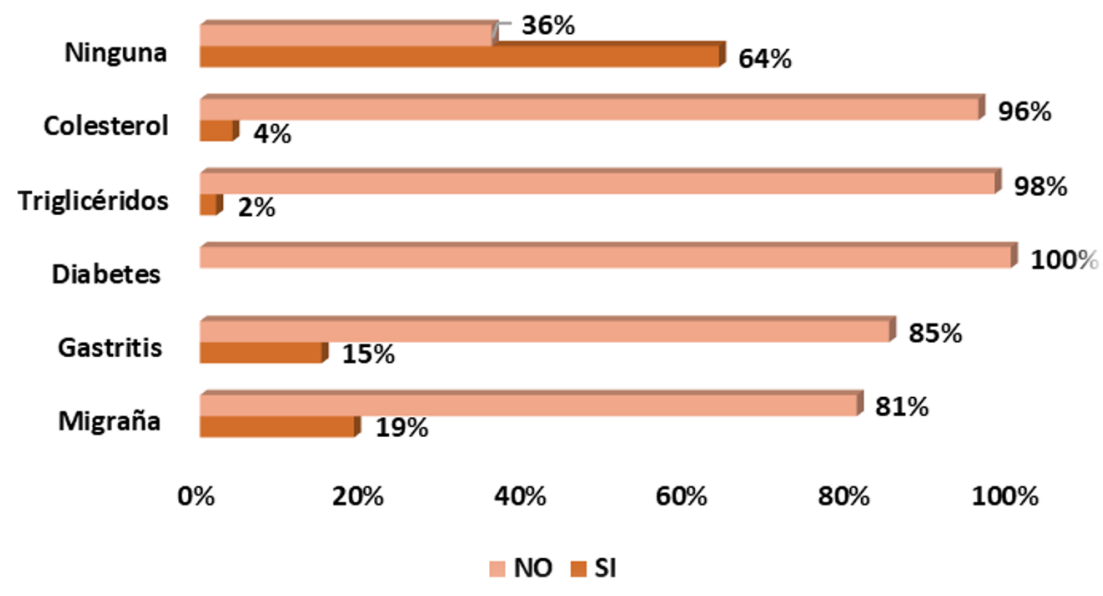

Fuente: Elaboración propia, a partir de cuestionarios aplicados a estudiantes

Teniendo en cuenta la población que afirmó poseer algunas de las enfermedades presentadas en la Gráfica 9, se indagó si consideraban la presencia de dichas enfermedades como consecuencia de sus hábitos alimenticios, obteniendo un 70\% como afirmativa y un 30\% como negativa. Por tanto, a pesar de ser pequeño el porcentaje de la población que afirma sufrir de alguna de las enfermedades anteriormente mencionadas, gran parte de ese grupo de personas lo relaciona con su alimentación, de tal forma se podría afirmar, de manera preliminar, que los estudiantes perciben que sus hábitos alimenticios son poco saludables y, además, les ha afectado los niveles de salud.

Gráfica 9. Porcentaje de estudiantes que relacionan las enfermedades con la alimentación

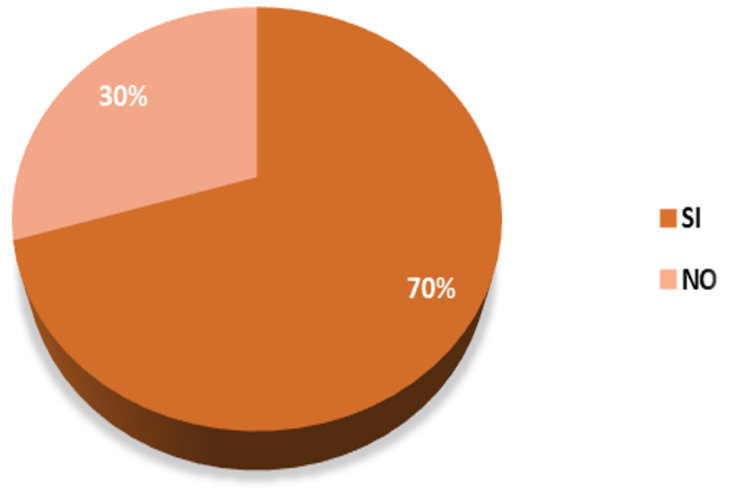

Fuente: Elaboración propia, a partir de cuestionarios aplicados a estudiantes

En la siguiente grafica se muestra los porcentajes de presencia de trastornos alimenticios, tales con la bulimia, anorexia y obesidad en la comunidad estudiantil de la Facultad de Ciencias Económicas. Los resultados arrojan que solo un 4\% 
han de tener obesidad, mientras que la anorexia y la bulimia se dan un $1 \%$. Por tanto, el $94 \%$ de los estudiantes no poseen ninguna de los trastornos mencionados.

Gráfica 10. Porcentaje de estudiantes que sufren trastornos alimenticios

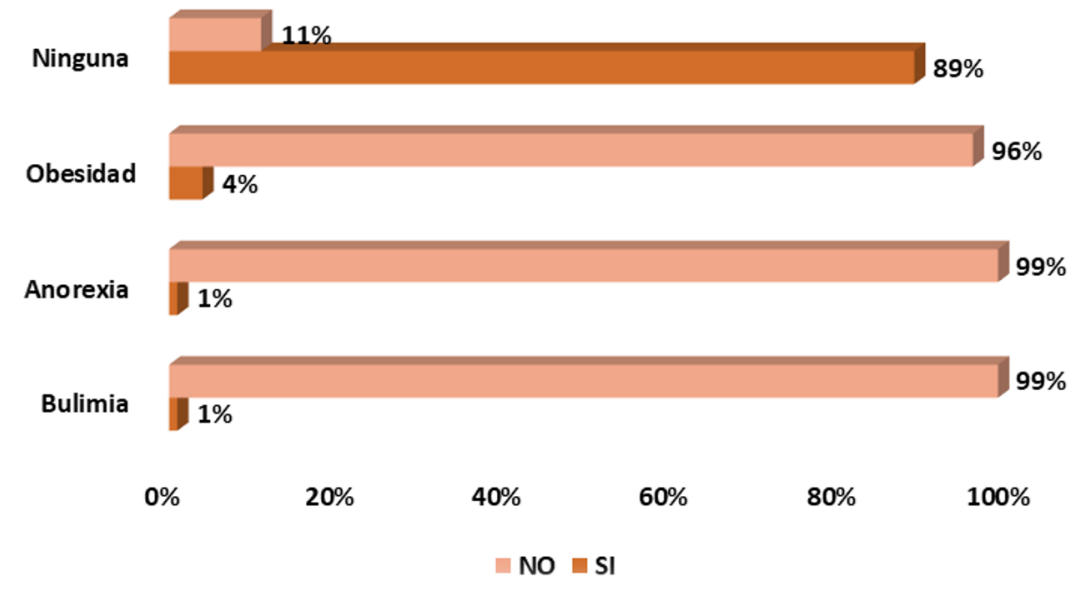

Fuente: Elaboración propia, a partir de cuestionarios aplicados a estudiantes

La población objeto de estudio en la presente investigación calificó, en una escala del 1 al 5, una serie de afirmaciones relacionadas con la percepción que tienen de estilos de vida y nivel de salud. Siendo así, el 59\% de la población encuestada considera importante llevar un ritmo de vida y una alimentación balanceada. Sin embargo, el porcentaje más representativo es de 38\% donde los estudiantes califican su nivel de salud en tres. A su vez, más del $50 \%$ de los estudiantes califican en un grado de 4 y 5 que reconocen las consecuencias de una buena o mala alimentación. Mientras que, un poco más del 50\% se concentra en un grado regular en cuanto a salud se refiere, lo que lleva a concluir que muchos estudiantes consideran no tener un muy buen nivel de salud (Tabla 3).

Tabla 3. Consideraciones de los estudiantes acerca de los estilos de vida saludables

\begin{tabular}{ccccc}
\hline $\begin{array}{c}\text { Escala de } \\
\text { calificación }\end{array}$ & $\begin{array}{c}\text { Considero importante } \\
\text { Ilevar un buen ritmo de } \\
\text { vida y una alimentación } \\
\text { balanceada }\end{array}$ & $\begin{array}{c}\text { Considero que } \\
\text { tengo una } \\
\text { alimentación } \\
\text { saludable }\end{array}$ & $\begin{array}{c}\text { Considero las } \\
\text { consecuencias de } \\
\text { una buena o mala } \\
\text { alimentación }\end{array}$ & $\begin{array}{c}\text { Considero } \\
\text { que tengo un } \\
\text { buen nivel de } \\
\text { salud }\end{array}$ \\
\hline 1 & $3,06 \%$ & $11 \%$ & $1,02 \%$ & $3,06 \%$ \\
\hline 2 & $7,14 \%$ & $29 \%$ & $8,16 \%$ & $16,33 \%$ \\
\hline 3 & $18,37 \%$ & $38 \%$ & $24,49 \%$ & $33,67 \%$ \\
\hline 5 & $12,24 \%$ & $16 \%$ & $36,73 \%$ & $29,59 \%$ \\
\hline
\end{tabular}

Fuente: Elaboración propia, a partir de cuestionarios aplicados a estudiantes

Teniendo en cuenta que según la muestra obtenida el 92\% del estudiantado consume alimentos dentro o cerca de la universidad de Cartagena (Gráfica 11), 
sede Piedra de Bolívar, se indagó percepción acerca de qué tan saludable consideran los alimentos ofertados en los establecimientos de venta de comida. Dentro del cuestionario se plantea un postulado que expresa lo siguiente ¿En qué grado considera usted que los alimentos que consume en los establecimientos de la Universidad de Cartagena o cerca de ella son saludables?, para el cual se condicionó con una escala del 1 al 5, donde 1 es poco saludable y 5 muy saludable, dicha pregunta arrojo que el $47 \%$ califica a los alimentos ofertados ni muy saludable ni poco saludables (Gráfica 12).

Gráfica 11. Estudiantes que consumen alimentos dentro o cerca de la Universidad (Sí consumen - No consumen)

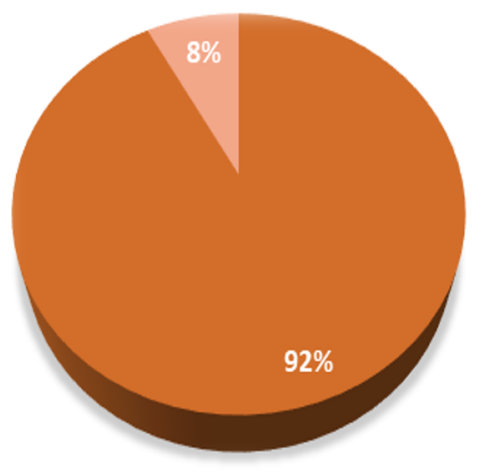

Fuente: Elaboración propia, a partir de cuestionarios aplicados a estudiantes

Gráfica 12. Percepción de los estudiantes acerca de los alimentos ofertados

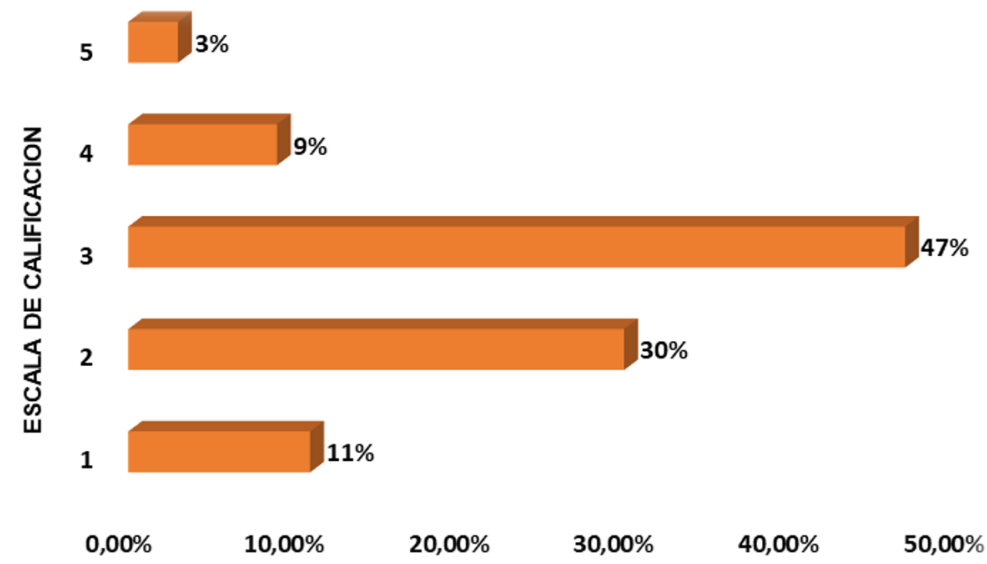

Fuente: Elaboración propia, a partir de cuestionarios aplicados a estudiantes

\section{CONCLUSIONES Y RECOMENDACIONES}

Esta investigación tuvo como objetivo conocer los hábitos alimenticios de los estudiantes de la Universidad de Cartagena, sede Piedra de Bolívar, así como la percepción que ellos tienen de la influencia de dichos hábitos sobre su salud. Para alcanzar dicho objetivo, se llevó a cabo una lista de chequeo aplicada a los 
establecimientos de la Universidad y alrededor de esta y una encuesta aplicada a los estudiantes de la universidad. Los resultados obtenidos muestran que el 92\% de los estudiantes de la población de estudio consumen alimentos al interior o en lugares aledaños a la Universidad, estas cifras representan un número preocupante pues la investigación reflejó una problemática con los establecimientos que ofertan algún tipo de alimento, y que está directamente relacionada con los hábitos alimenticios de los estudiantes.

La problemática se centra en la poca oferta y asequibilidad de alimentos que contribuyan a una dieta saludable. Según la lista de chequeo realizada a los establecimientos alimentarios, solo el $50 \%$ de los establecimientos ofertan alimentos definidos como "saludable" y tienen un promedio de precios de $\$ 7.000$, lo que resulta un poco costoso para un estudiante universitario de estrato 1,2 o 3 y que ingiere alimentos en la Universidad más de 2 veces por semana (aproximadamente $70 \%$ de la población de estudio).

El otro 50\% de los establecimientos ofertan alimentos definidos como "poco saludables", de este porcentaje, el $60 \%$ oferta frituras, este es el alimento más demandado por los estudiantes y esto debido, principalmente, a la variedad y el precio del producto (en promedio, \$1.600). Este precio, contrario al de los alimentos saludables, es mucho más asequible para los estudiantes y, aunque la mayoría de los alumnos saben que este alimento es perjudicial para la salud, de igual manera lo consumen porque es lo que pueden adquirir con su presupuesto económico.

Con respecto a la encuesta y percepción de los estudiantes, un 78\% de los encuestados considera que tiene malos hábitos alimenticios, lo que es entendible por los alimentos ofertados; un 37\% tiene enfermedades comunes en los jóvenes universitarios (migraña, gastritis, diabetes, triglicéridos, colesterol u otra) y, de todas estas enfermedades, un 65\% de la población considera que están estrechamente relacionadas con los hábitos alimenticios que estos tienen.

Por último, y como respuesta a nuestro objetivo de proponer a los estudiantes sugerencias que puedan ser aplicables y les ayude a mejorar sus hábitos alimenticios, se plantean los siguientes consejos apoyados en información proporcionada por la Organización Panamericana de la Salud (OPS):

- Es importante consumir una variedad de alimentos pues ingerir el mismo tipo de alimentos resulta poco beneficioso al cuerpo pues ningún alimento contiene la cantidad necesaria de nutrientes para que el cuerpo este saludable, por eso se sugiere una dieta que combine carnes, verduras, frutas y granos. 
- Aumentar el consumo de agua es importante que no se olvide de la hidratación del cuerpo y para esto nada mejor que el agua, además de ayudar con la digestión y mejorar el sistema inmunológico.

- Una recomendación muy importante es reducir el uso de ciertas grasas y aceites, estas no aportan contenido nutricional al cuerpo, aunque no es bueno dejarlas del todo si es recomendable reducir su consumo para así evitar enfermedades que se desarrollan al ingerirlas frecuentemente.

- Llevar consigo alimentos que no se oferten o que se consiguen con poca frecuencia al interior y/o exterior de la Universidad y es necesario consumir diariamente por su contenido nutricional.

\section{REFERENCIAS}

Arroyo, A., Gutiérrez, D., Narváez, A., Ramos, P., Maza, F., (2019). Hábitos alimenticios y sus efectos en la salud de los estudiantes de la Facultad de Ciencias Económicas de la Universidad de Cartagena. Mimeo

Burriel, F. Serrano, R. Vico, C. Milla, M. García, M. (2013). Hábitos alimentarios y evaluación nutricional en una población universitaria.

Cervera Burriel, F., Serrano Urrea, R., Vico García, C., Milla Tobarra, M., \& García Meseguer, M. J. (2013). Hábitos alimentarios y evaluación nutricional en una población universitaria. Nutrición Hospitalaria, 28(2), 438-446. https://doi.org/10.3305/nh.2013.28.2.6303

Del Águila R. (s.f.) Promoción de estilos de vida saludable y prevención de enfermedades crónicas en el adulto mayor. Organización Panamericana de la Salud. https://www.doktuz.co/wikidoks/prevencion/estilos-de-vida-saludable.html

FAO (2018). Transformar la alimentación y la agricultura para alcanzar los ODS. 20 acciones interconectadas para guiar a los encargados de adoptar decisiones (FAO). $\quad$ http://www.fao.org/publications/transforming-food-agriculture-toachieve-sdg/es/

ICBF (2018). ENSIN: Encuesta Nacional de Situación Nutricional 2015. Bogotá. https://www.asivamosensalud.org/actualidad/presentacion-ensin-2015

OMS (2016). Obesidad y sobrepeso. https://www.who.int/es/news-room/factsheets/detail/obesity-and-overweight

Universidad de Cartagena. (2017). Reseña histórica. http://www.unicartagena.edu.co/universidad/historia

Universidad de Cartagena. (2018). Boletín estadístico 2018. http://www.unicartagena.edu.co/inicio/transparencia-y-acceso-a-lainformacion/metas-e-indicadores/boletin-estadistico 